\title{
Numerical Simulation during Short-Shot Water-Assisted Injection Molding Based on the Overflow Cavity for Short-Glass Fiber-Reinforced Polypropylene
}

\author{
Zhong Yu, ${ }^{1,2}$ He-Sheng Liu $\mathbb{D}^{1,3}$ Tang-Qing Kuang, ${ }^{4}$ Xing-Yuan Huang, ${ }^{1}$ Wei Zhang, \\ Zhong-Shi Chen, ${ }^{1}$ and Kai Zhang ${ }^{2}$ \\ ${ }^{1}$ Polymer Processing Laboratory, Nanchang University, Nanchang 330031, China \\ ${ }^{2}$ Jiangxi Province Key Laboratory of Polymer Preparation and Processing, Shangrao Normal University, Shangrao 334001, China \\ ${ }^{3}$ School of Chemical Biology and Materials, East China University of Technology, Nanchang 330013, China \\ ${ }^{4}$ School of Mechanical \& Electrical Engineering, East China Jiaotong University, Nanchang 330013, China
}

Correspondence should be addressed to He-Sheng Liu; hsliu@vip.163.com

Received 18 July 2019; Revised 31 December 2019; Accepted 4 February 2020; Published 5 May 2020

Academic Editor: Huamin Zhou

Copyright (c) 2020 Zhong Yu et al. This is an open access article distributed under the Creative Commons Attribution License, which permits unrestricted use, distribution, and reproduction in any medium, provided the original work is properly cited.

\begin{abstract}
Compared with water penetration condition of short-shot water-assisted injection molding with or without overflow cavity, it can be known from theory and common knowledge that short-shot water-assisted injection molding with overflow cavity has many advantages, such as it can save materials and energy. Then, the effects of melt short shot size, water injection delay time, melt temperature and water injection pressure on the penetration of water after penetration, and the orientation distribution of short fibers during water-assisted injection molding of the overflow cavity short-shot method were studied. It is found that the melt short shot size had the greatest influence on it, followed by water injection pressure, water injection delay time, and finally, melt temperature. With the increase of the melt short shot size, the thickness of the residual wall of the whole main cavity becomes thinner, the orientation of short fiber along the melt flow direction becomes higher, and the degree of fiber orientation changes becomes lower. In the front half of the main cavity, with the decrease of water injection pressure, the delay time of water injection, and the melt temperature, in the front part of the main cavity, the residual wall thickness becomes thinner, the fiber orientation along the melt flow direction becomes lower, and the fiber orientation changes degree becomes higher; in the latter half of the main cavity, the influence of the water penetration and the orientation distribution of short fibers along the melt flow direction are not significant.
\end{abstract}

\section{Introduction}

Fluid-assisted injection molding is a new injection molding technique that uses high-pressure fluid to push molten polymer forward to form a hollow or partially hollow product. It consists of gas-assisted injection molding and water-assisted injection molding. Because the gas-assisted system was more mature than the water-assisted system, gas-assisted injection molding first entered the researcher's field of vision. With the development of science and technology, the technology of the water-assisted system has gradually improved, and waterassisted injection molding has gradually entered the field of researchers. Compared with gas, water has the advantages of high thermal conductivity, specific heat capacity, and recyclability, so water-assisted injection molding can shorten the molding cycle, reduce the production cost, and improve the inner surface smoothness, and so on $[1,2]$. Therefore, in recent years, water-assisted injection molding has been widely concerned.

In water-assisted injection molding, according to whether the molten polymer is fully filled with the main cavity before high-pressure water injection, it can be divided into short-shot water-assisted injection molding and overflow water-assisted injection molding. Whether it is short-shot 
or overflow molding, researchers focus on the influence of process parameters on macrostructure such as the water penetration length and residual wall thickness [3]. For example, Huang and Deng [4] studied the influence of process parameters on the water penetration length and residual wall thickness of PP curved pipe products by using a single-factor method. Zhang et al. [5] and Zhang et al. [6] studied the influence of relevant process parameters on the residual wall thickness of water-assisted injection molded products. Polynkin et al. [7] simulated the influence of water injection pressure on the residual wall thickness of water-assisted injection molded circular pipe products. Pudpong et al. [8] used Moldflow to simulate the influence of process parameters on the residual wall thickness of overflow water-assisted injection molding PP products by an overflow method. Park et al. [9] found that the residual wall thickness of the workpiece decreases with the increase of polymer melt temperature and water injection pressure through experiments and theories. Tangqing et al. and Tang-Qing et al. $[10,11]$ studied the influence of the water pressure and water delay time on the residual wall thickness of water-assisted coinjection molding by experiment. Recently, the researchers also conducted correlation studies on the microstructure and performance control of water-assisted injection molding products by changing process parameters and physical parameters [12]. For example, Wang et al. [13] prepared polypropylene/acrylonitrile-styrene copolymer products with four mass ratios through different water injection pressure and melt temperature and studied the formation mechanism of transverse crystal. Xianhu et al. $[14,15]$ studied the effect of HDPE with different molecular weights on the crystal morphology of water-assisted injection molded products. Haiying et al. [16] studied the effect of process parameters on the short fiber circumferential orientation by experiments and theories. In the literature investigation, there are many researches on shortshot water-assisted injection molding without the overflow cavity. However, there are few researches on the short-shot water-assisted injection molding based on the overflow cavity.

In this paper, the numerical calculation is carried out based the short-shot water-assisted injection molding with and without overflow cavity. The residual wall thickness and water penetration length were compared when the short-shot molding with and without the overflow cavity is used under the same process parameters. It is proved by common sense and theory that short injection with overflow cavity can save more materials than short injection without overflow cavity. Then, the influence of process parameters on the residual wall thickness and fiber orientation distribution degree of short fiberreinforced PP products by short-shot water-assisted injection molding with the overflow cavity is analyzed. At the same time, it also broadens the basic theoretical and experimental research content of short-shot water-assisted injection molding.

\section{Numerical Research Method}

The short-shot water-assisted injection molding process based on the overflow cavity, which first fills short glass fiber-reinforced polymer composite melt into the main cavity, and then injects high-pressure water into the main cavity to push the melt forward to the overflow cavity, which finally keeps pressure and cooling. Due to the intervention of highpressure water, the whole process involves many process parameters. The melt short shot size, water injection delay time, melt temperature, and water injection pressure are the main research objects in the calculation.

\section{Mathematical Model and Assumption}

Short-shot water-assisted injection molding based on the overflow cavity involves both short fiber-reinforced polymer composites with viscoelastic melt and high-pressure water for Newtonian fluids. In order to make the calculation convergence and the calculation process conform to the actual molding conditions, the mathematical model is simplified as follows: (1) the density, heat capacity, and thermal conductivity in the fluid remain unchanged; (2) no slip between the melt and the mold wall; (3) ignoring surface tension, gravity, inertial force, and body force; and (4) not considering the phase transition enthalpy during melt crystallization. Therefore, the threedimensional nonisothermal flow behavior governing equation is obtained as follows:

Continuity equation:

$$
\frac{\partial \rho}{\partial t}+\nabla \cdot \rho \boldsymbol{u}=0
$$

Momentum equation:

$$
\frac{\partial}{\partial t}(\rho \boldsymbol{u})+\nabla \cdot(\rho \boldsymbol{u} \boldsymbol{u}+\boldsymbol{\tau})=-\nabla p+\rho \boldsymbol{g}
$$

Energy equation:

$$
\rho C_{p}\left(\frac{\partial T}{\partial t}+\boldsymbol{u} \cdot \nabla T\right)=\nabla \cdot(k \nabla T)+\eta \dot{\gamma}^{2}
$$

where $\boldsymbol{u}$ is the velocity vector, $T$ is temperature, $t$ is time, $p$ is pressure, $\boldsymbol{\tau}$ is stress tensor, $\boldsymbol{g}$ is acceleration vector of gravity, $\rho$ is density, $\eta$ is viscosity, $k$ is thermal conductivity, $C_{p}$ is specific heat, and $\dot{\gamma}$ is shear rate.

In order to describe the rheological properties of polymer melt in the mold flow channel, one of the classical constitutive equations reflecting the short fiber-reinforced polymer composites, the White-Metzner constitutive equation, is selected:

$$
\begin{aligned}
\boldsymbol{\tau}+\lambda\left(\frac{\partial \boldsymbol{\tau}}{\partial t}+\boldsymbol{u}\right. & \left.\cdot \nabla \boldsymbol{\tau}-\nabla \boldsymbol{u}^{\mathrm{T}} \cdot \boldsymbol{\tau}-\boldsymbol{\tau} \cdot \nabla \boldsymbol{u}\right)=\eta\left(\nabla \boldsymbol{u}+\nabla \boldsymbol{u}^{\mathrm{T}}\right) \\
\lambda(T, \dot{\gamma}) & =\frac{\eta(T, \dot{\gamma})}{G} \\
\eta & =\frac{\eta_{0}}{1+\left(\eta_{0} \dot{\gamma} / \tau^{*}\right)^{1-n}} \\
\eta_{0} & =\mathrm{D}_{1} \exp \left(\frac{-\mathrm{A}_{1}\left(T-T_{c}\right)}{\mathrm{A}_{2}+\left(T-T_{c}\right)}\right) \\
T_{c} & =\mathrm{D}_{2}+\mathrm{D}_{3} p \\
\mathrm{~A}_{2} & =\tilde{\mathrm{A}}_{2}+\mathrm{D}_{3} p
\end{aligned}
$$


TABLE 1: Characteristics of polypropylene composites (10\% by weight of short fibers).

\begin{tabular}{|c|c|}
\hline Thermal conductivity & $k=0.1776 \mathrm{~W} /(\mathrm{m} \cdot \mathrm{K})$ \\
\hline Specific heat capacity & $C_{P}=2700 \mathrm{~J} /(\mathrm{kg} \cdot \mathrm{K})$ \\
\hline White-Metzner constitutive equation parameters & $\begin{array}{c}n=0.20426, \tau^{*}=33086.7 \mathrm{~Pa}, D_{1}=4.58 \mathrm{E}+20 \mathrm{~Pa} \cdot \mathrm{s}, \\
D_{2}=263.15 \mathrm{~K}, D_{3}=0 \mathrm{~K} / \mathrm{Pa}, A_{1}=40.388, \tilde{A}_{2}=51.6 \mathrm{~K} \text {, and } G=2.1 \mathrm{E}+05 \mathrm{~Pa}\end{array}$ \\
\hline Fiber orientation model iARD-PRP parameters & $C_{I}=0.005, C_{M}=0$, ansd $\alpha=0.7$ \\
\hline
\end{tabular}

where $T$ is the melt temperature, $G$ is shear modulus, $\eta_{0}$ is zero shear viscosity, $\tau^{*}$ is the parameter of the transition region between the zero shear rate and the power law region of the viscosity curve, $n$ is the power law index, and $\mathrm{D}_{1}, \mathrm{D}_{2}, \mathrm{D}_{3}, \mathrm{~A}_{1}$, and $\tilde{\mathrm{A}}_{2}$ are all the material constants.

In fiber orientation simulation, orientation probability distribution function and orientation tensor are often used to describe fiber orientation. The orientation distribution function can describe the fiber orientation state directly, but the complexity limits its use. The orientation tensor describes the fiber orientation state in the form of the matrix of the orientation distribution function. In previous studies, the standard Folgar-Tucker orientation equation combined with the fluid dynamics model of the IRD model was a useful method to theoretically determine the orientation of isotropic fibers in the concentrated suspension, but when quantitatively compared with the relevant experimental observations, the equation showed the inaccuracy of excessive prediction. Recently, the Phelps-Tucker anisotropic rotational diffusion (ARD) model has demonstrated the ability to deal with the orientation of primary anisotropic fibers; however, ARD tensors dependent on HD tensors are generally difficult to apply. In this paper, the recently improved iARD-RPR orientation model [17] is adopted, which not only considers the interaction between fibers but also considers the rotation resistance of fibers in three-dimensional space. Its expression is as follows:

$$
\begin{aligned}
\dot{\mathbf{A}} & =\dot{\mathbf{A}}^{\mathrm{HD}}+\dot{\mathbf{A}}^{\mathrm{iARD}}\left(C_{I}, C_{M}\right)+\dot{\mathbf{A}}^{\mathrm{RPR}}(\alpha), \\
\dot{\mathbf{A}}^{\mathrm{HD}} & =(\mathbf{W} \cdot \mathbf{A}-\mathbf{A} \cdot \mathbf{W})+\xi\left(\mathbf{D} \cdot \mathbf{A}+\mathbf{A} \cdot \mathbf{D}-2 \mathbf{A}_{4}: \mathbf{D}\right),
\end{aligned}
$$

where $\dot{\mathbf{A}}^{\text {iARD }}$ has two available parameters: the fiber-fiber interaction parameter $C_{I}$ and the fiber-matrix interaction parameter $C_{M} ; \dot{\mathrm{A}}^{\mathrm{RPR}}$ has one parameter $\alpha$, which is meant to slow down the response rate of the fiber orientation.

In this paper, the material selected was a short fiberreinforced polypropylene (SGFPP) with a mass content of $10 \%$ short fibers, which was produced by LyondellBasell Industries. According to the equations mentioned above, the physical parameters involved are shown in Table 1.

3.1. Melt front tracking. A volume percent function is introduced to describe the melt front position and the evolution of fluid penetration with respect to time. Here, $f=0$ is defined as the fluid phase, $f=1$ as the plastic melt phase, whereas the melt front is located in the $0<f<1$ unit. The increase in the volume percent fraction from the kinematics process is governed by the following advection equation:

$$
\frac{\partial f}{\partial t}+\nabla \cdot(\boldsymbol{u} f)=0
$$

where $f$ is the volume percent fraction; $\boldsymbol{u}$ is the velocity vector.

In this paper, based on the White-Metzner constitutive equation describing the short fiber-reinforced polymer composite and the short fiber orientation model iRAD-PRP, a mathematical model was constructed based on three major equations of fluid dynamics. Using the finite volume method and the melt front tracking method, three fluid equations, constitutive equations, and fiber orientation models were calculated based on the given process parameters and physical parameters.

\section{Model Structure and Process Parameters}

Short-shot water-assisted injection molding with the overflow cavity is different from the general short-shot waterassisted injection molding by adding an overflow cavity. Its model consists mainly of the main cavity, overflow cavity, melt injection nozzle, and water injection nozzle. Its main structural parameters: the main cavity size is $\Phi 16 * 245$, the overflow cavity diameter is $\Phi 10$. Compared with short-shot water-assisted injection molding based on the overflow cavity, there is no overflow cavity for the short-shot waterassisted injection molding without the overflow cavity. The schematic diagrams and physical figure are shown in Figure 1 and Figure 2, respectively.

In this paper, firstly, the residual wall thickness and water penetration length were compared when the short-shot molding without the overflow cavity and the short-shot molding with the overflow cavity are used under the same process parameters. The following, the influence of process parameters on the residual wall thickness and fiber orientation distribution degree of short fiber-reinforced PP products by short-shot water-assisted injection molding with the overflow cavity, are further analyzed; the process parameters studied are shown in Table 2.

\section{Materials and Experiments}

5.1. Materials. In the experiments, short glass fiberreinforced polyethylene (SGFPP, Grade Hostacom SB224-1, LyondellBasell Industries, Germany) was used. The materials were $10 \%$ reinforced by the mass content of short glass fiber. 


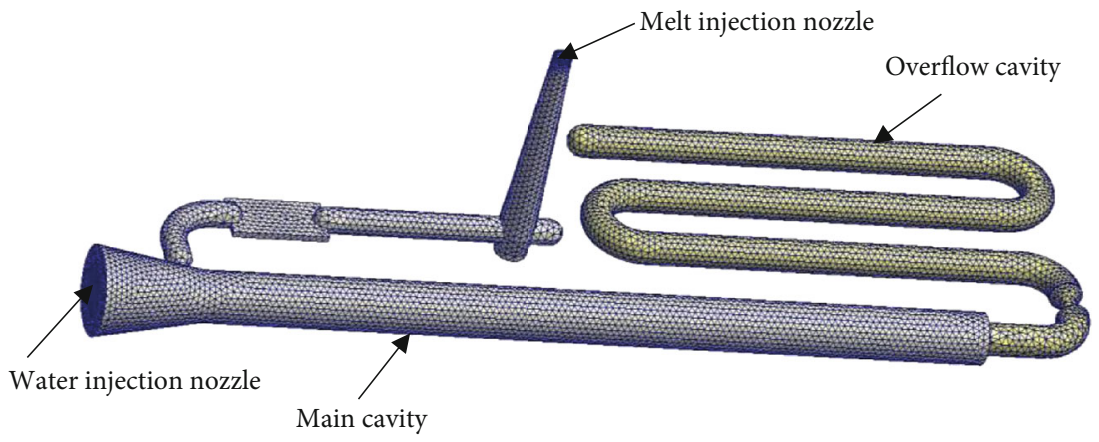

FIGURE 1: The schematic diagrams.

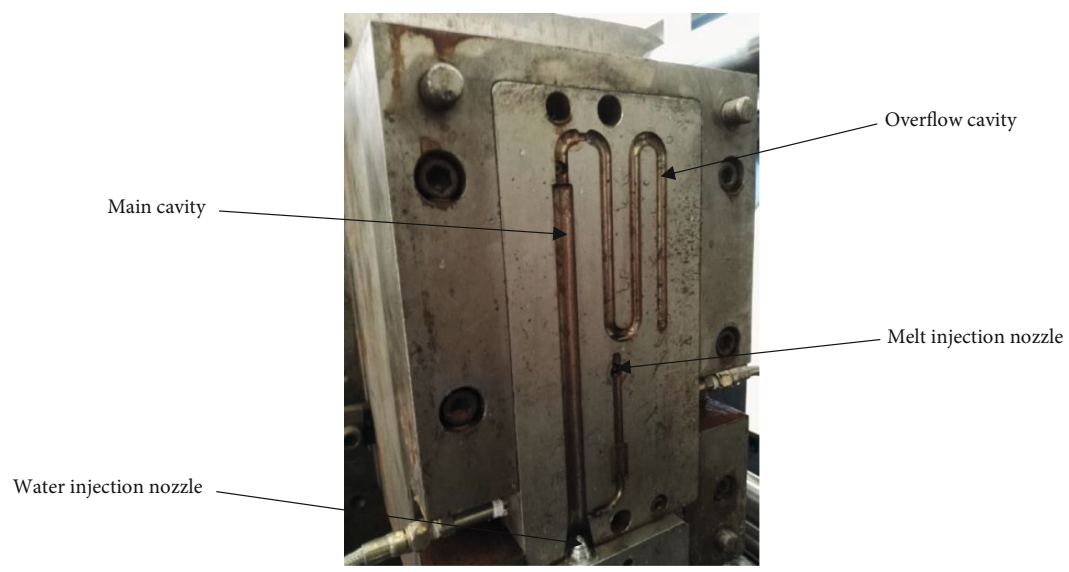

Figure 2: The physical figure.

TABLE 2: The process parameters.

\begin{tabular}{lccc}
\hline Melt short shot size $(\%)$ & Water injection delay time $(\mathrm{s})$ & Melt temperature $\left({ }^{\circ} \mathrm{C}\right)$ & Water injection pressure $(\mathrm{MPa})$ \\
\hline 70 & 0 & 210 & 4 \\
75 & $\mathbf{1}$ & $\mathbf{2 3 0}$ & 6 \\
80 & 3 & 250 & $\mathbf{8}$ \\
85 & 5 & 270 & 10 \\
\hline
\end{tabular}

Note: The numerical bolding in the table is the basic parameter to investigate the melt short shot size, water injection delay time, melt temperature, and water injection pressure.

Its flow index MFI $(230,2.16)=7 \mathrm{~g} / 10 \mathrm{~min}$, and its density was $0.9 \mathrm{~g} / \mathrm{cc}$.

5.2. Experiments. The experimental platform is mainly composed of injection molding machine, water injection system, and mold, as shown in Figure 3. The experiment adopts a single-factor experiment, in which the injection molding machine mainly implements melt short shot size and melt temperature, and the water injection system mainly implements injection water injection delay time and water injection pressure.

\subsection{Theoretical and Experimental Data Processing}

5.3.1. Residual Wall Thickness and Its Percentage. The residual wall thickness of the product refers to the radial thickness of the melt after filling, and the percentage of residual wall

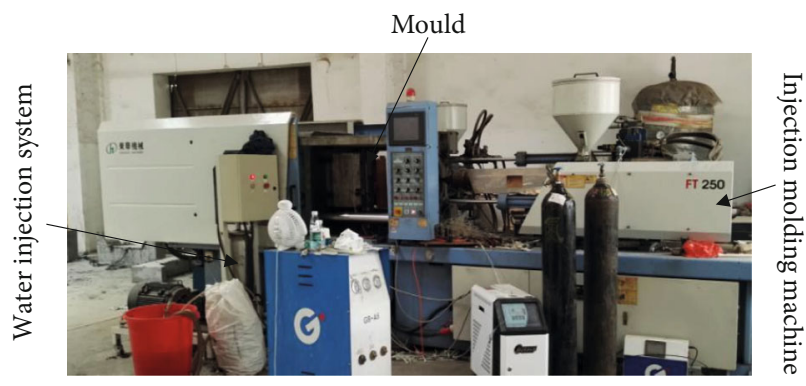

Figure 3: The experimental platform.

thickness refers to the ratio of the residual wall thickness to the main cavity diameter. The larger the percentage of the residual wall thickness, the larger the cross-section of melt penetrated by water, and vice versa. 

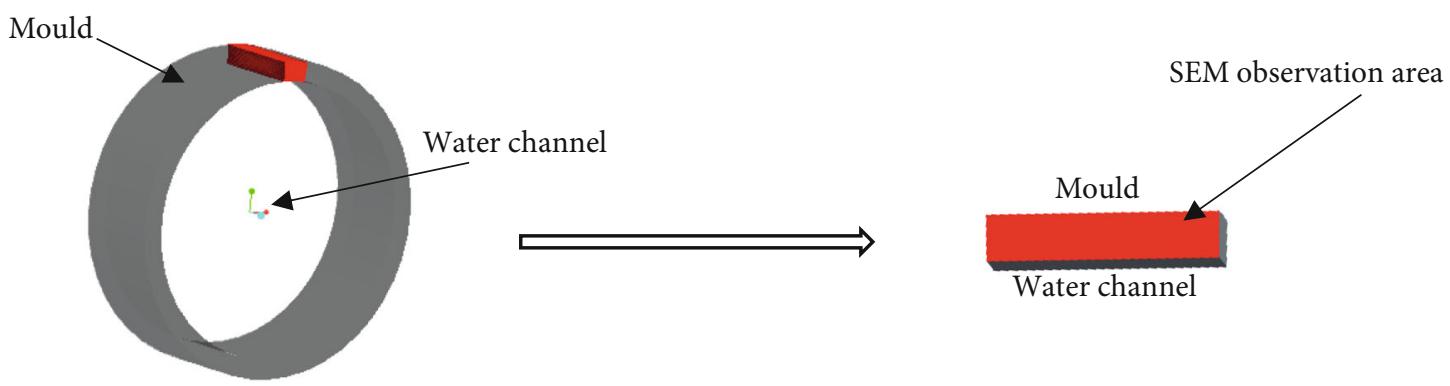

Figure 4: Schematic diagram of SEM sample sampling position and detection area.

5.3.2. Fiber Orientation. Fiber orientation refers to the orientation value of the fiber along the melt flow direction. The more the orientation value fluctuates or the more chaotic the orientation value is, the greater the orientation change degree is. In this paper, the main cavity is divided into three sections on average. At the end of each section, a $5 \mathrm{~mm}$ ring is cut out (Figure 4) and then placed in a liquid nitrogen container for half an hour cooling and brittle fracture treatment. Finally, the brittle section is sliced and placed in a carrier platform with conductive adhesive for gold spraying (Figure 5) and scanning electron microscope (Figure 6).

\section{Results and Analysis}

Figure 7 shows the water penetration of the two waterassisted injection moldings along the melt flow direction at the melt short shot size of $75 \%$, the water injection delay time of $1 \mathrm{~s}$, the melt temperature of $230^{\circ} \mathrm{C}$, and the water injection pressure of $8 \mathrm{MPa}$. It can be seen from the figure that the main cavity is completely penetrated by water during shortshot water-assisted injection molding with the overflow cavity, while the main cavity is penetrated by water more than half during short-shot water-assisted injection molding without the overflow cavity.

The reason is that in the short-shot water-assisted injection molding without the overflow cavity, the volume of the main cavity corresponds to the sum of the volume of the melt penetrated by water and the volume of the residual wall thickness, and the more the melt is penetrated by water, the smaller the residual wall thickness. On the contrary, the less the melt is penetrated by water, the larger the residual wall thickness. In short-shot water-assisted injection molding with the overflow cavity, although the volume of the main cavity is equal to the sum of the volume of the melt penetrated by water and the volume of the residual wall thickness, the overflow cavity is connected with the main cavity causes the excess melt to gradually flow into the overflow cavity during the process of the melt penetrated by water. Under the same process parameters, compared with the short-shot water-assisted injection molding without the overflow cavity, when the short-shot water-assisted injection molding with the overflow cavity is used, the melt is longer penetrated by water and the residual wall thickness is thinner; the short-shot water-assisted injection molding with the overflow cavity saves more material or energy, such as melt short shot size.

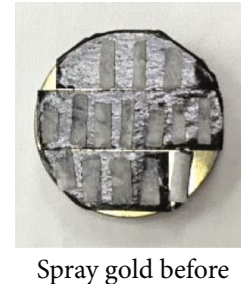

(a)

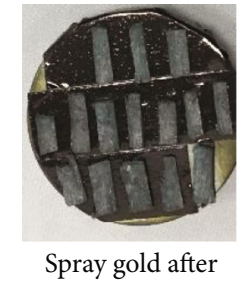

(b)
Figure 5: Experimental test sample.

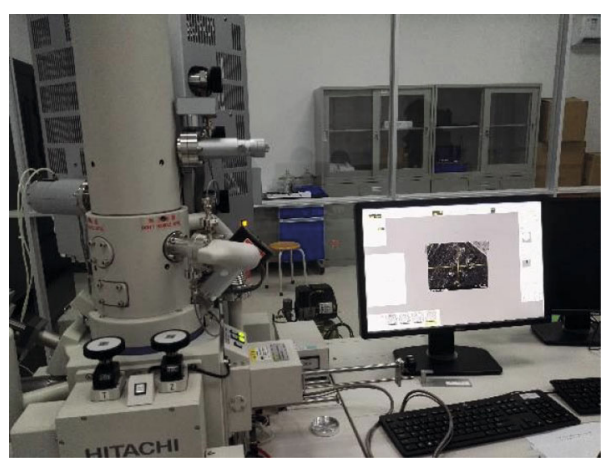

FIgURE 6: SEM observation.

Therefore, both in theory and in common sense, the short-shot water-assisted injection molding with the overflow cavity is more advantageous than that without the overflow cavity. Subsequently, the influence of different process parameters on water penetration and fiber orientation distribution along the melt flow direction will be analyzed during the short-shot water-assisted injection molding with the overflow cavity.

6.1. Water Penetration. Figure 8 shows the residual wall thickness percentage along the melt flow direction when the melt short shot size of the short-shot water-assisted injection molding with the overflow cavity is $70 \%, 75 \%$, $80 \%$, and $85 \%$, respectively; other process parameters are the basic parameters. As can be seen from the figure, as the melt short shot size increases, residual wall thickness percentage increases, and residual wall thickness is thickening. At a distance from the water injection port to the end of the main cavity, as the melt short shot size is smaller, the residual wall thickness percentage is flatter and smaller, and the residual wall thickness is more uniform. The reason is 


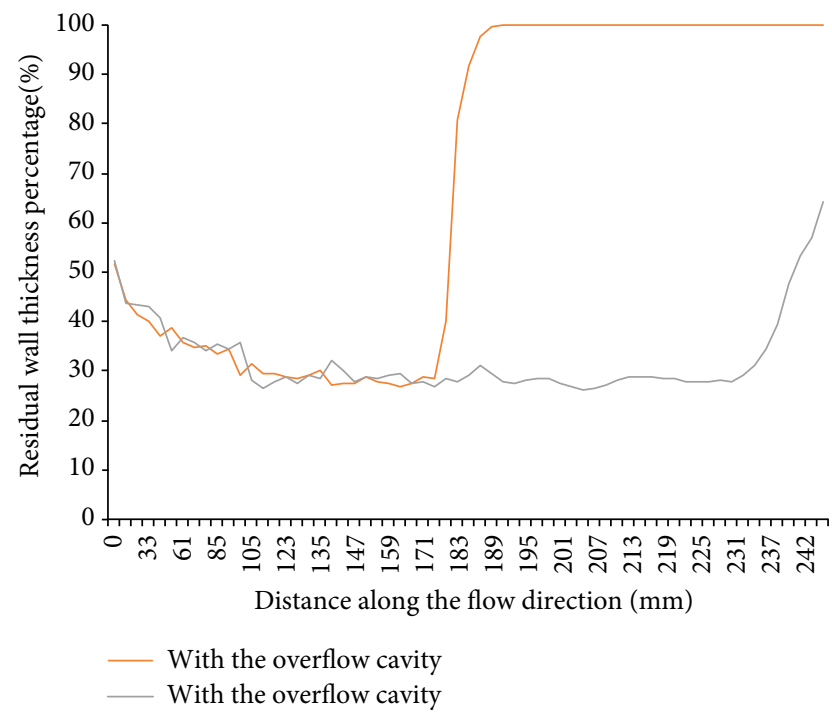

Figure 7: Water penetration of two short-shot water-assisted injection molding at the melt injection volume of $75 \%$.

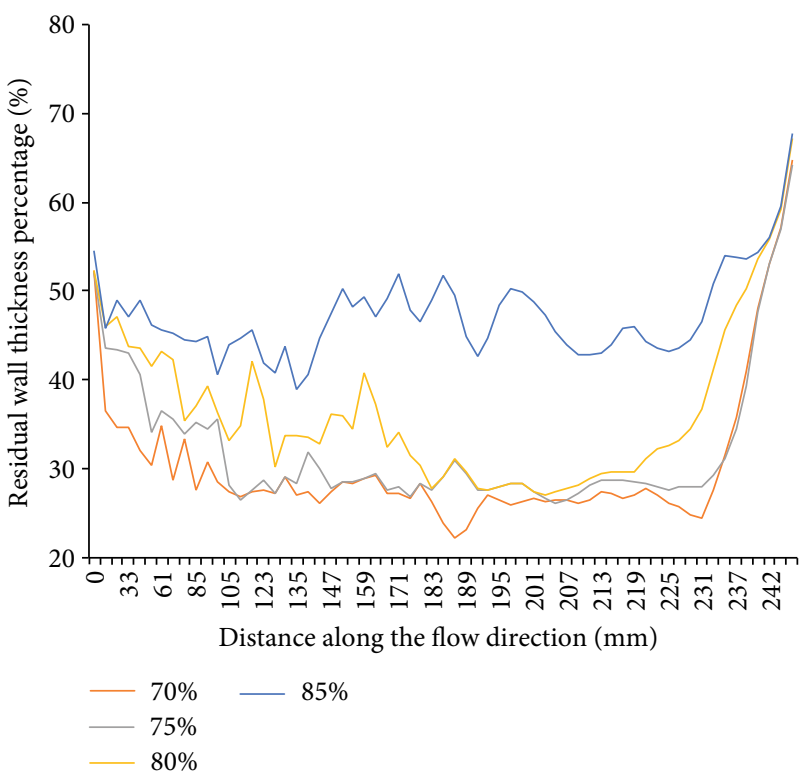

FIGURE 8: Effect of melt short shot size on water penetration.

that as the melt short shot size increases, the resistance of water penetrates increases, and the longer the cooling time of the melt that is not penetrated by the water at the low temperature mold wall, the greater the effect.

Figure 9 shows that the residual wall thickness percentage along the melt flow direction when the injection delay time of the short-shot water-assisted injection molding with the overflow cavity is $0 \mathrm{~s}, 1 \mathrm{~s}, 3 \mathrm{~s}$, and $5 \mathrm{~s}$, respectively; other process parameters are the basic parameters. It can be seen from the figure that with the longer of water injection delay time, residual wall thickness percentage increases in the front half of the main cavity, and residual wall thickness percentage in the latter half is basically the same. The reason is that

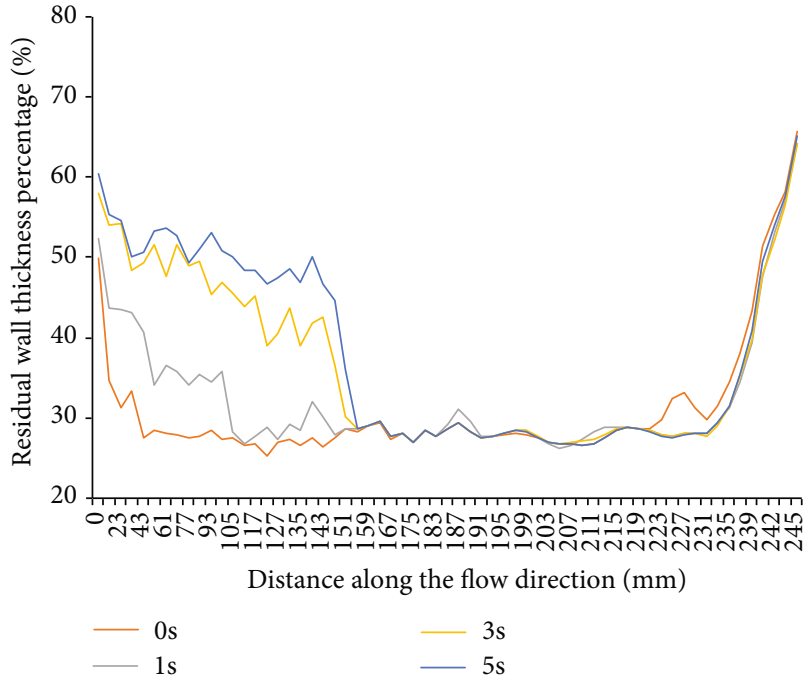

FIGURE 9: Effect of water injection delay time on water penetration.

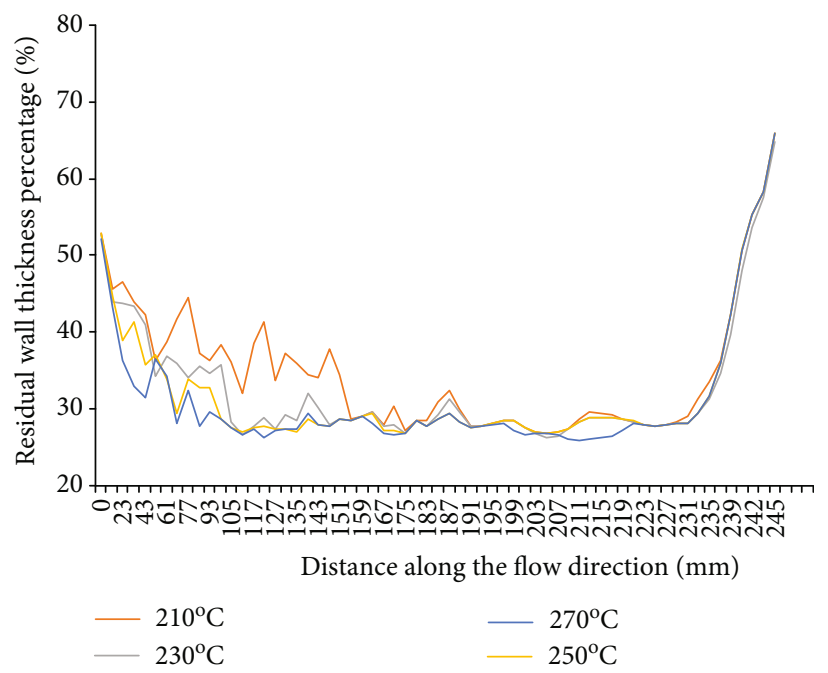

FIGURE 10: Effect of melt temperature on water penetration.

before the high-pressure water is injected, the hightemperature melt mainly stays in the front half of the main cavity, and as the cooling time of low-temperature mold wall becomes longer, the thickness of solidified layer becomes larger. After the high-pressure water is injected, the melt of the front half of the main cavity is pushed into the overflow cavity through the latter half of the main cavity. In this process, the residual wall thickness of the latter half of the main cavity is determined by the mold surface and the water cooling period. This period is very short, resulting in substantially the same percentage of residual wall thickness in the latter half. In addition, it can be seen that when the water injection time is $0 \mathrm{~s}$, residual wall thickness percentage is the flattest along the melt flow direction.

Figure 10 shows that the residual wall thickness percentage along the melt flow direction when the melt temperature of the short-shot water-assisted injection molding with the 


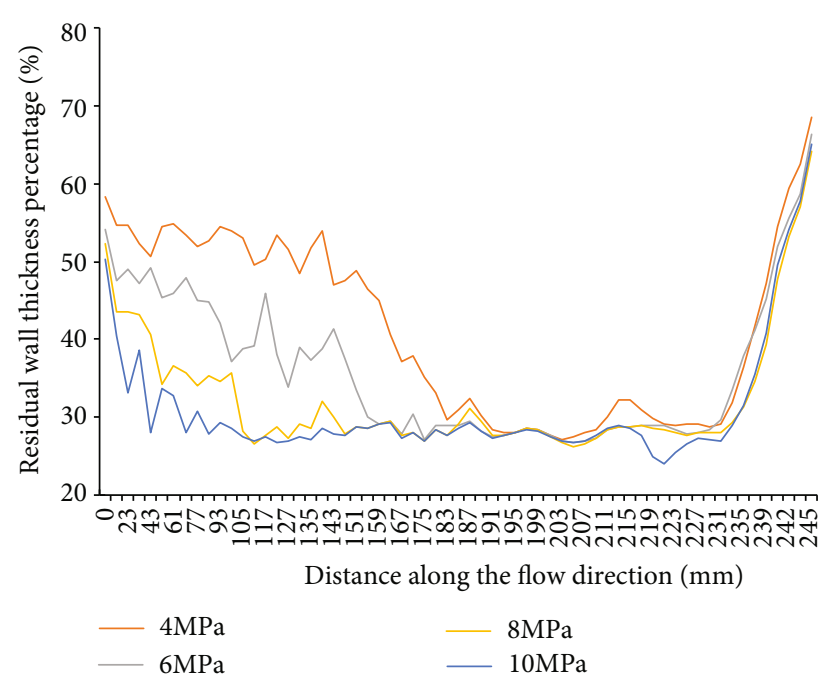

FIGURE 11: Effect of water injection pressure on water penetration.

overflow cavity is $210^{\circ} \mathrm{C}, 230^{\circ} \mathrm{C}, 250^{\circ} \mathrm{C}$, and $270^{\circ} \mathrm{C}$, respectively; other process parameters are the basic parameters. According to the figure, as the melt temperature increases, residual wall thickness percentage decreases relatively, but not significant. The reason is that as the melt temperature increases, the melt viscosity decreases, the resistance of high-pressure water pushing melt decreases, and residual wall thickness percentage also decreases.

Figure 11 shows that the residual wall thickness percentage along the melt flow direction when the water injection pressure of the short-shot water-assisted injection molding with the overflow cavity is $4 \mathrm{MPa}, 6 \mathrm{MPa}$, $8 \mathrm{MPa}$, and $10 \mathrm{MPa}$, respectively; other process parameters are the basic parameters. It can be seen from the figure that as the water injection pressure increases, residual wall thickness percentage decreases, and the distribution of residual wall thickness is flatter along the melt flow direction, and the percentage of residual wall thickness in the latter half of the main cavity is almost the same. The reason is that as the water injection pressure increases, the water diameter becomes large when the high-pressure water enters from the water injection nozzle, thereby pushing the water penetration cross-section to be larger. In the latter half of the main cavity, the melt here is driven by the high-pressure water in the first half of the main cavity and continues to be pushed into the overflow cavity by the high-pressure water. During this process, the melt is pushed by the high-pressure water for a short time, resulting in a shorter time for the melt to be cooled by water and mold wall.

6.2. Degree of Fiber Orientation Distribution. Figure 12 shows that the fiber orientation distribution along the melt flow direction when the melt short shot size of the short-shot water-assisted injection molding with the overflow cavity is $70 \%, 75 \%, 80 \%$, and $85 \%$, respectively; other process parameters are the basic parameters. As can be seen from the figure, as the melt short shot size increases, the fiber orientation is lower and the degree of change in the fiber orientation distribution is higher along the melt flow direction. According to the characteristics of short fiber-reinforced polymer waterassisted injection molding products, the products are divided into a wall layer, core layer, and water channel layer along the direction of thickness. The fiber at the wall layer and water channel layer has a high orientation along the melt flow direction and a low degree of change, while the fiber at the core layer has a random orientation along the melt flow direction and a high degree of change. The reasons are as follows: as can be seen from Figure 8, as the melt short shot size increases, residual wall thickness percentage increases, and residual wall thickness is thickening. The thickening of residual wall thickness means that the smaller the percentage of wall thickness of the wall layer and water channel layer, the higher the percentage of core layer, resulting in the lower orientation and higher degree of change of fiber along the melt flow direction.

Figure 13 shows that the fiber orientation distribution along the melt flow direction when the water injection delay time of the short-shot water-assisted injection molding with the overflow cavity is $0 \mathrm{~s}, 1 \mathrm{~s}, 3 \mathrm{~s}$, and $5 \mathrm{~s}$, respectively; other process parameters are the basic parameters. As can be seen from the figure, in the front half of the main cavity, as the water injection delay time is prolonged, the fiber orientation is lower and the degree of the fiber orientation change is higher along the melt flow direction. In the latter half of the main cavity, the change in the water injection delay time has little effect on it. The reasons are as follows: as can be seen from Figure 9, with the longer of water injection delay time, residual wall thickness percentage increases in the front half of the main cavity, and residual wall thickness percentage in the latter half is basically the same. With the extension of water injection delay time, the percentage of residual wall thickness in the front half of the main cavity increases and the residual wall thickness thickens, resulting in the percentage of core layer in the wall thickness increases and the lower orientation and higher change degree of the fibers in the front half of the main cavity along the melt flow direction.

Figure 14 shows that the fiber orientation distribution along the melt flow direction when the melt temperature of the short-shot water-assisted injection molding with the overflow cavity is $210^{\circ} \mathrm{C}, 230^{\circ} \mathrm{C}, 250^{\circ} \mathrm{C}$, and $270^{\circ} \mathrm{C}$, respectively; other process parameters are the basic parameters. As can be seen from the figure, in the front half of the main cavity, as the melt temperature increases, the fiber orientation is higher and the degree of change in the fiber orientation distribution is lower along the melt flow direction. In the latter half of the main cavity, the change in the melt temperature has little effect on it. The reasons are as follows: as can be seen from Figure 10, as the melt temperature increases, the residual wall thickness percentage decreases in the front half of the main cavity, and the residual wall thickness percentage in the latter half is basically the same, but not significant in the main cavity. With the increase of melt temperature, the percentage of residual wall thickness in the front half of the main cavity increases and the residual wall thickness thickens, resulting in the percentage of core layer in the wall thickness increases 


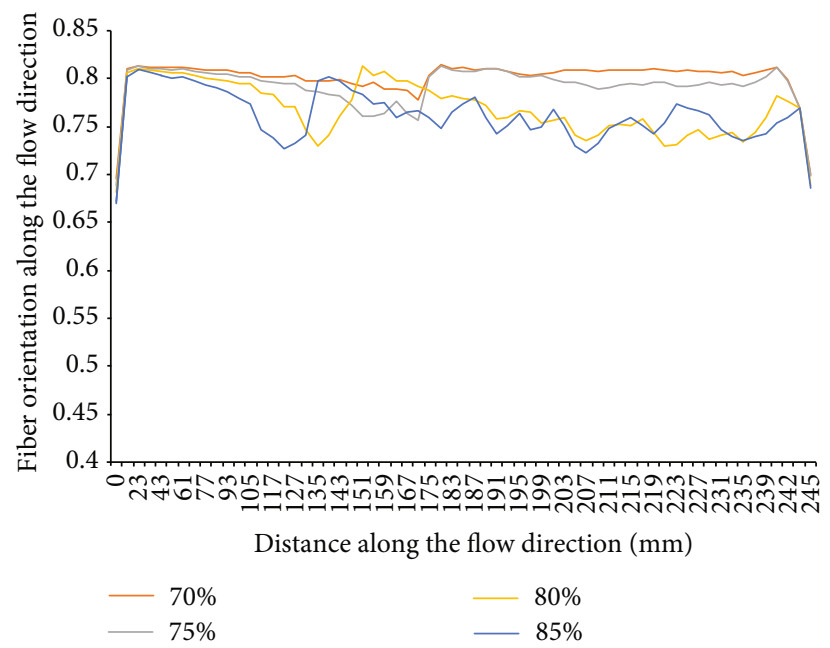

(a) Numerical analysis of water injection delay time on fiber orientation
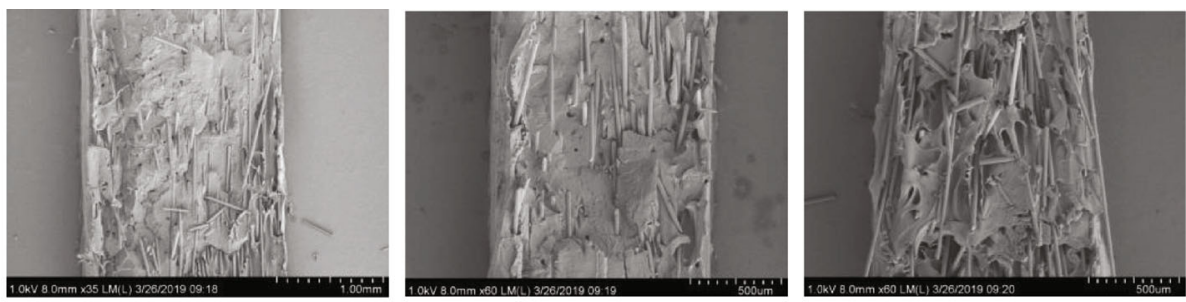

$70 \%$
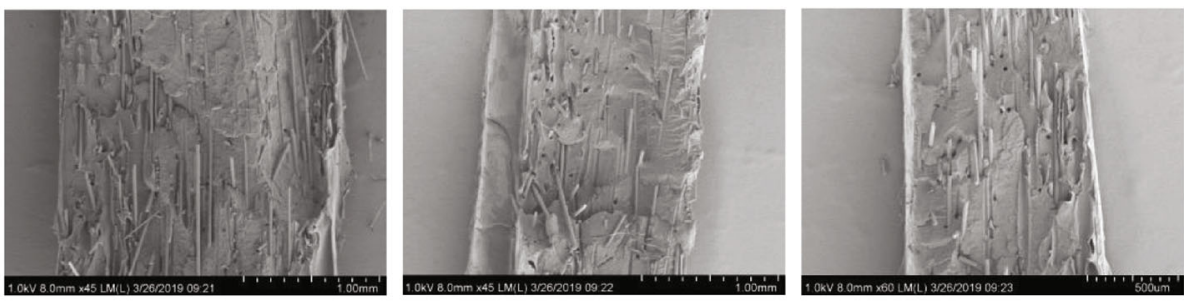

$75 \%$
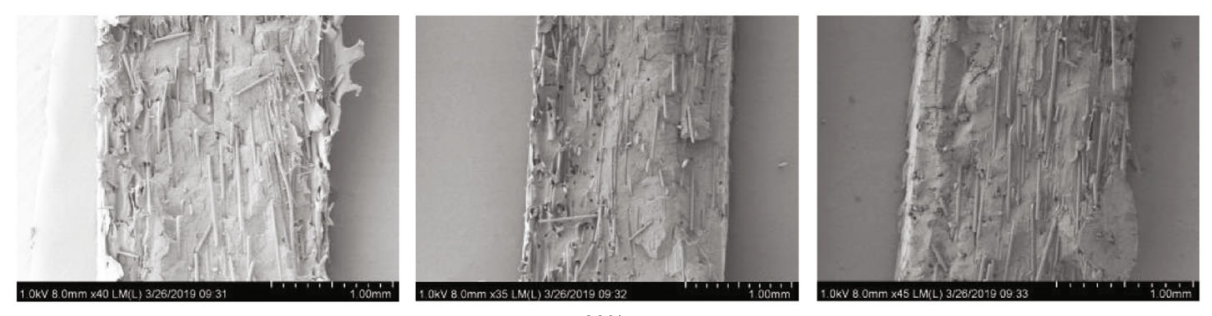

$80 \%$
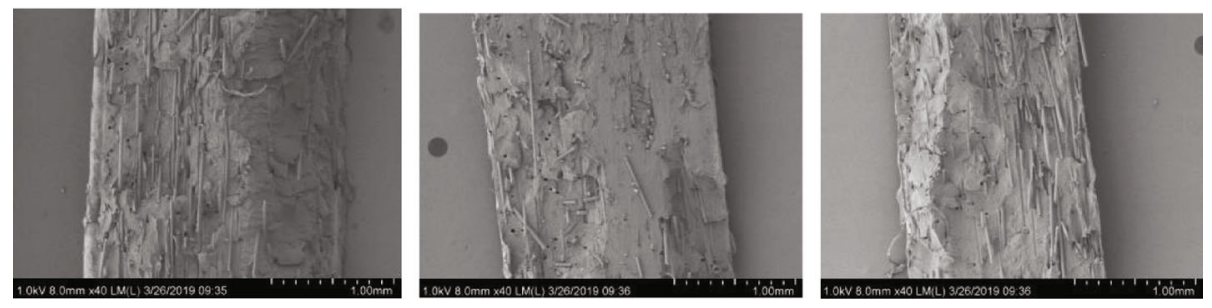

(b) SEM image of fiber orientation, the left side of each picture is the mold wall, the right side is the channel, and the three pictures of each process parameter are the near water end, the middle end, and the far water end, respectively, from left to right (the result of $0 \mathrm{~s}$ the same as the result of $1 \mathrm{~s}$ )

Figure 12: Effect of melt short shot size on fiber orientation distribution along the melt flow direction. 


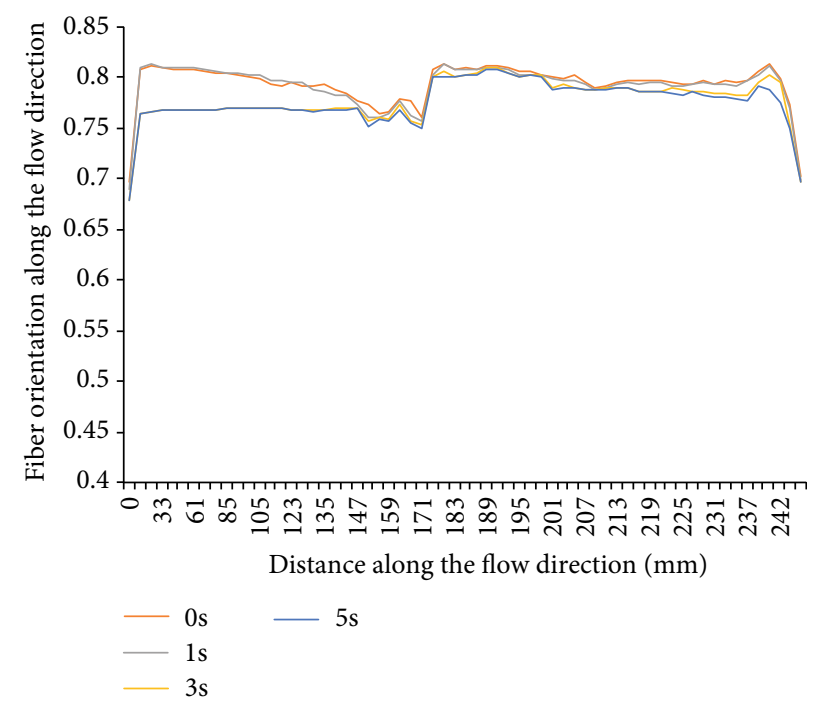

(a) numerical analysis of water injection delay time on fiber orientation
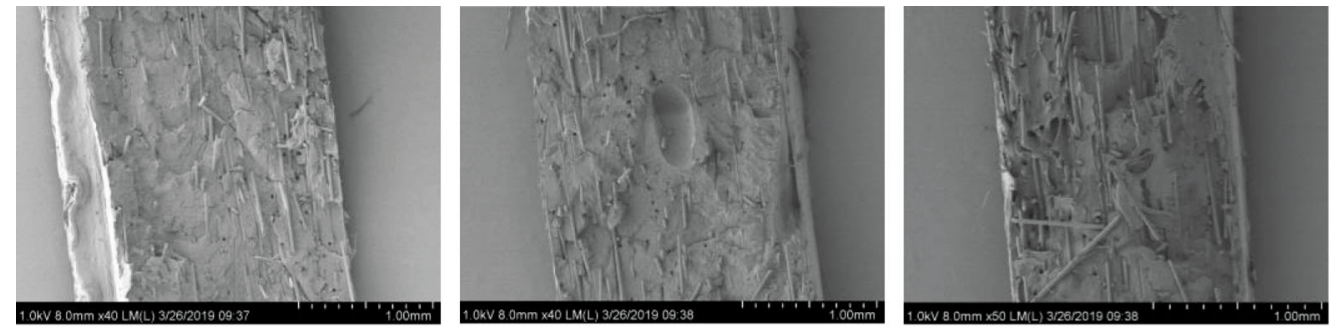

$1 \mathrm{~s}$
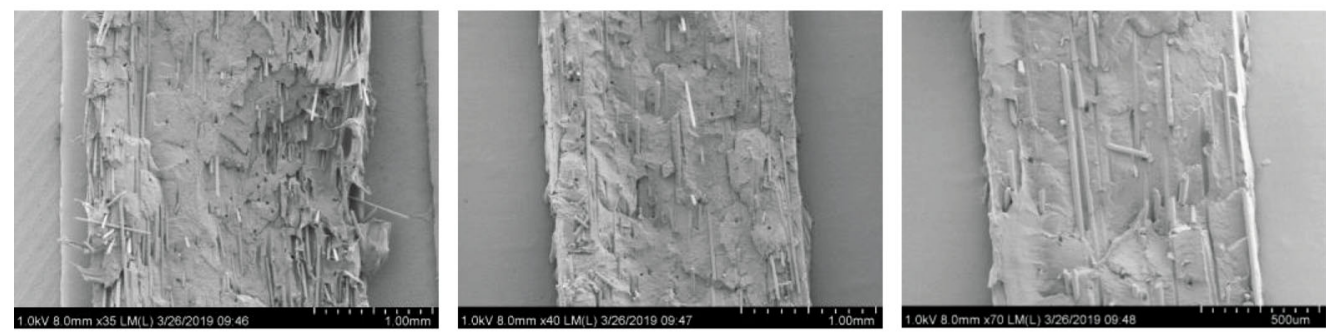

3s
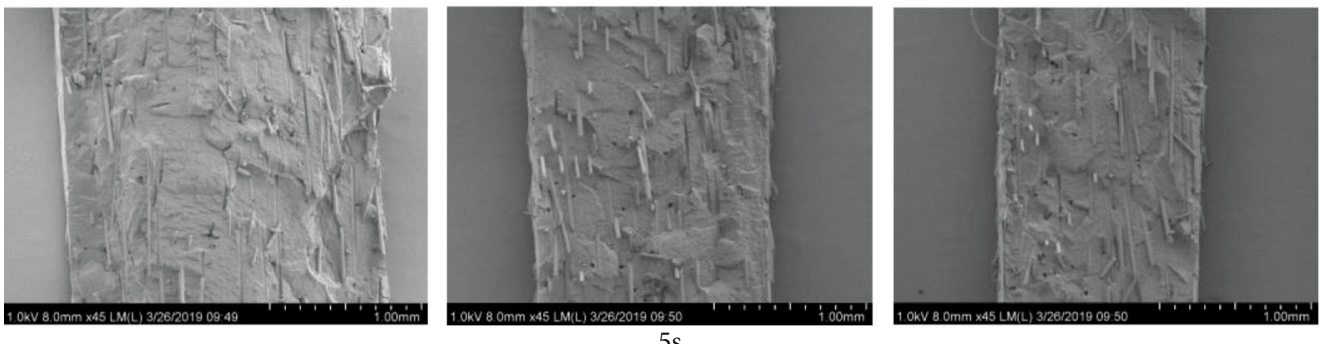

(b) SEM image of fiber orientation, the left side of each picture is the mold wall, the right side is the channel, and the three pictures of each process parameter are the near water end, the middle end and the far water end respectively from left to right (the result of $0 \mathrm{~s}$ the same as the result of $1 \mathrm{~s}$ )

FIGURE 13: Effect of water injection delay time on fiber orientation distribution along the melt flow direction.

and the lower orientation and higher change degree of the fibers in the front half of the main cavity along the melt flow direction.

Figure 15 shows that the fiber orientation distribution along the flow direction when the water injection pressure of the short-shot water-assisted injection molding with the overflow cavity is $4 \mathrm{MPa}, 6 \mathrm{MPa}$, $8 \mathrm{MPa}$, and $10 \mathrm{MPa}$, respectively; other process parameters are the basic parameters. As can be seen from the figure, in the front half of the main cavity, as the water injection pressure increases, the fiber orientation is higher and the degree of change in the fiber orientation distribution is lower along the melt flow 


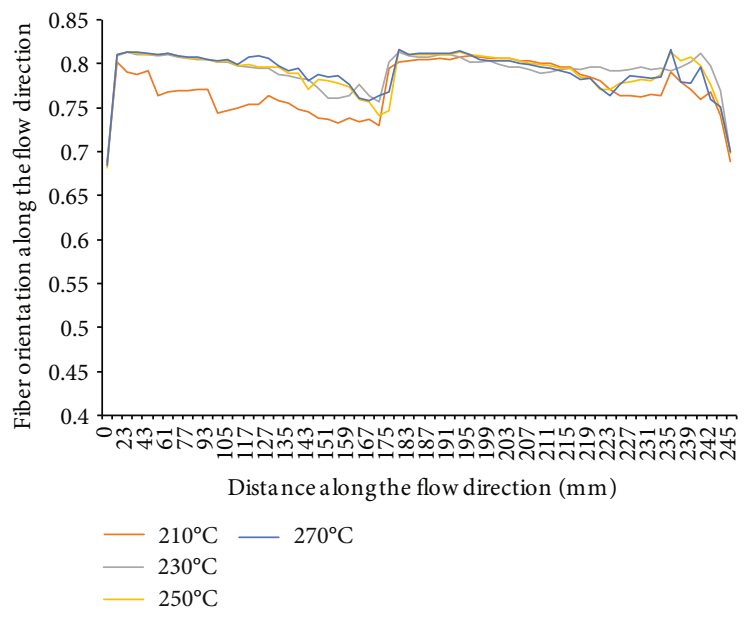

(a) Numerical analysis of melt temperature on fiber orientation
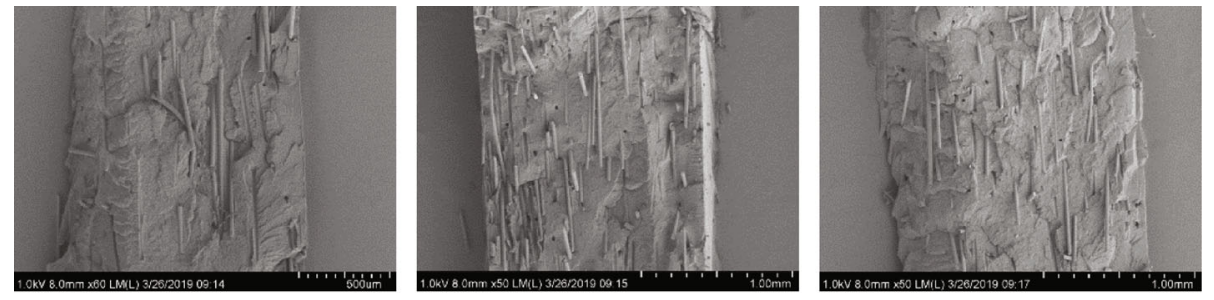

$210^{\circ} \mathrm{C}$
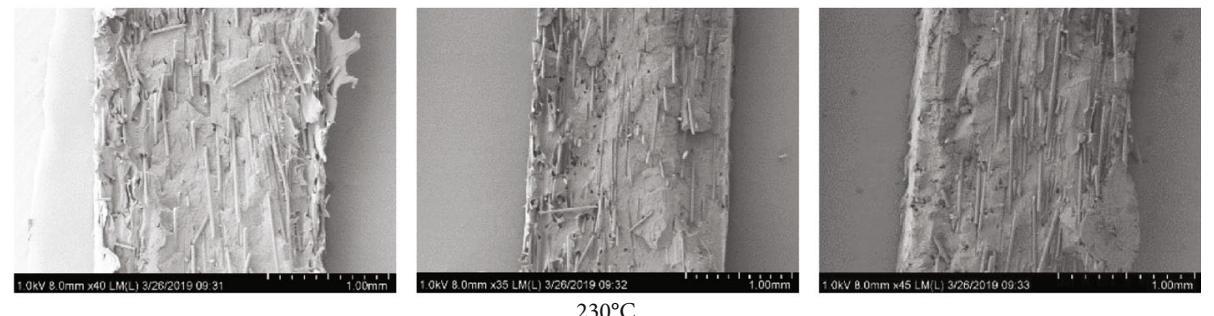

$230^{\circ} \mathrm{C}$
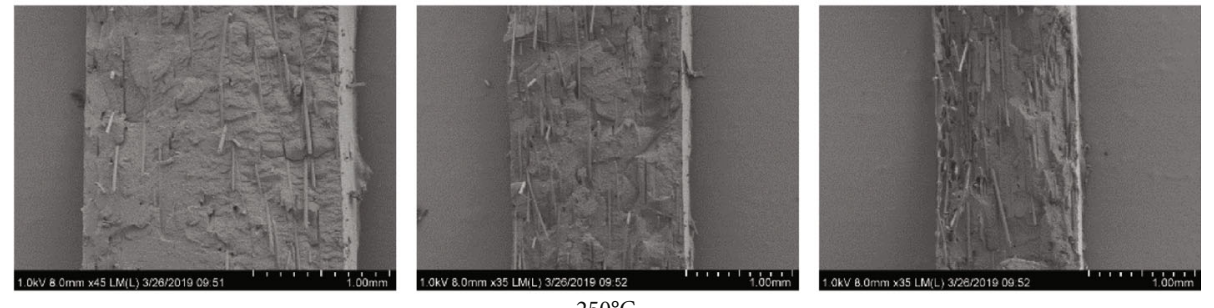

$250^{\circ} \mathrm{C}$
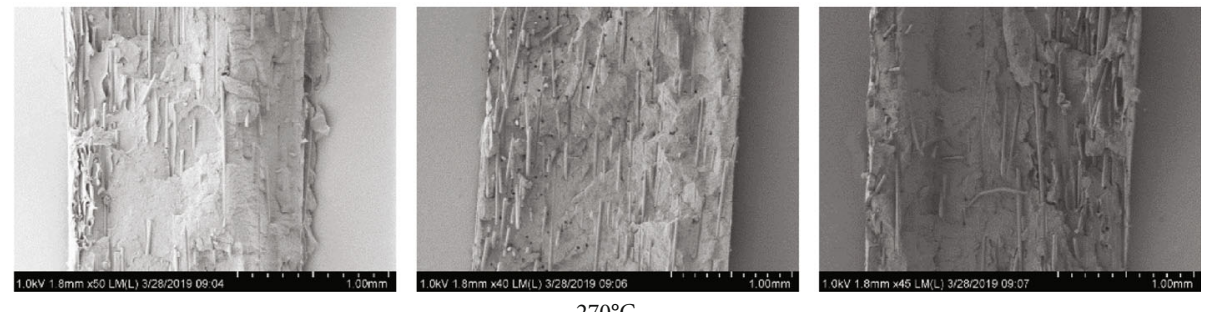

$270^{\circ} \mathrm{C}$

(b) SEM image of fiber orientation, the left side of each picture is the mold wall, the right side is the channel, and the three pictures of each process parameter are the near water end, the middle end and the far water end, respectively, from left to right

FIGURE 14: Effect of melt temperature on fiber orientation distribution along the melt flow direction. 


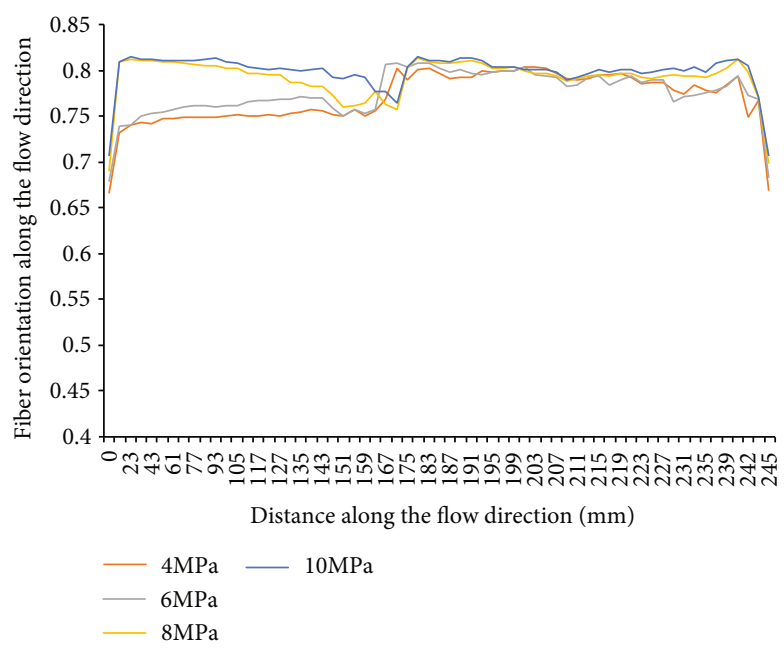

(a) Numerical analysis of water injection pressure on fiber orientation
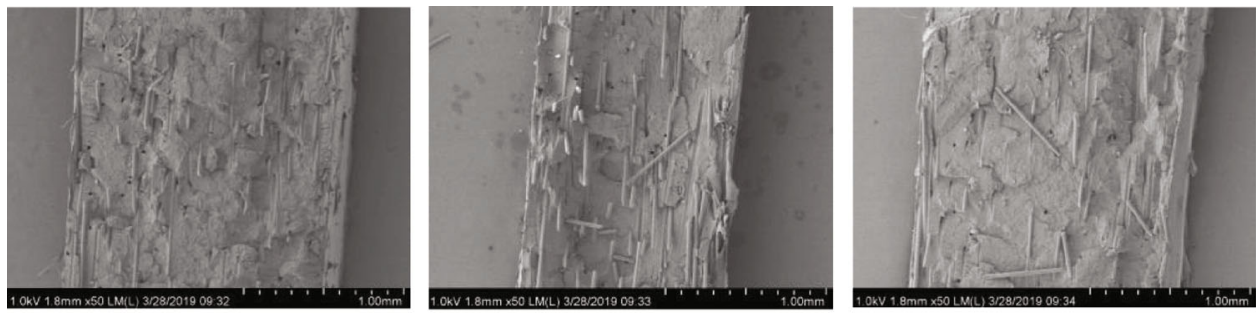

$4 \mathrm{MPa}$
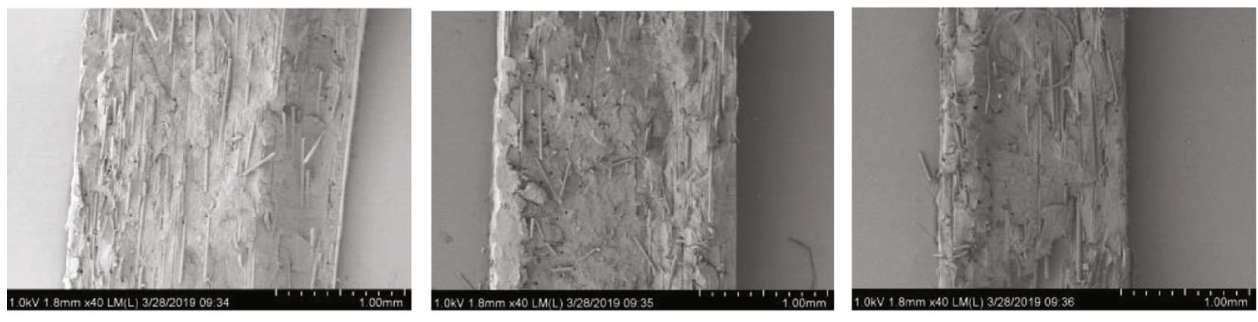

$6 \mathrm{MPa}$
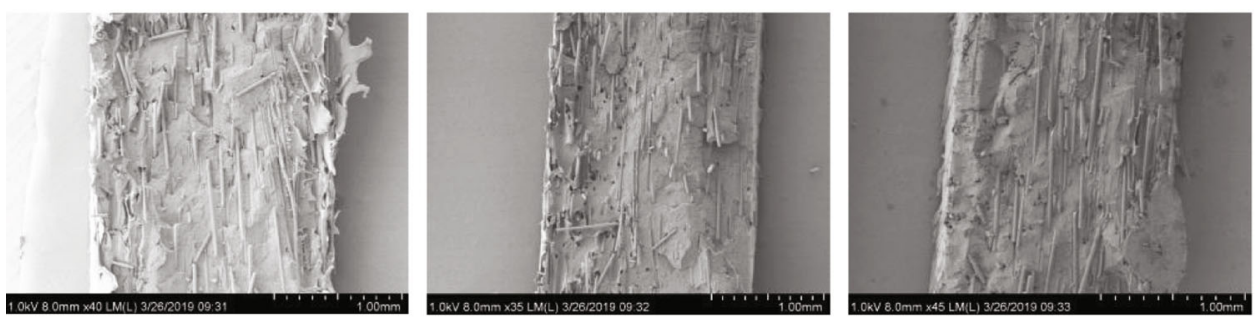

$8 \mathrm{MPa}$
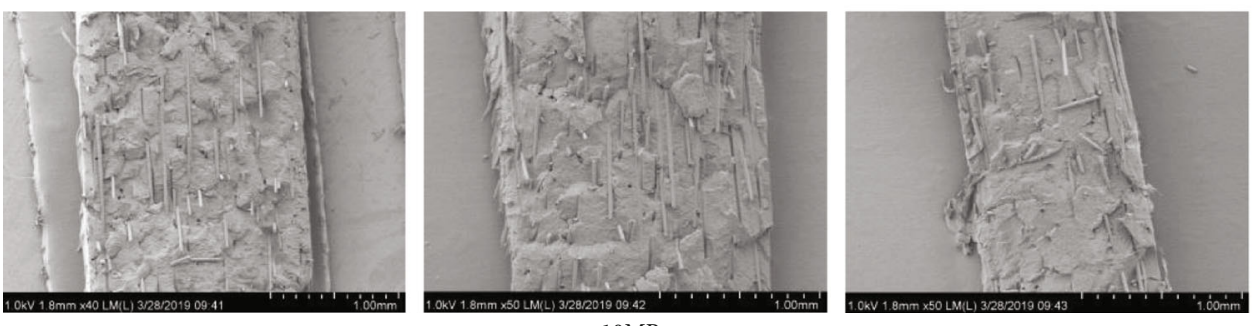

$10 \mathrm{MPa}$

(b) SEM image of fiber orientation, the left side of each picture is the mold wall, the right side is the channel, and the three pictures of each process parameter are the near water end, the middle end and the far water end, respectively, from left to right

FIGURE 15: Effect of water injection pressure on fiber orientation distribution along the melt flow direction. 
direction. In the latter half of the main cavity, the change in the melt temperature has little effect on it. The reasons are as follows: as can be seen from Figure 11, as the water injection pressure increases, residual wall thickness percentage decreases in the front half of the main cavity, and residual wall thickness percentage in the latter half is basically the same. With the increase of water injection pressure, the percentage of residual wall thickness in the front half of the main cavity decreases and the residual wall thickness thins, resulting in the percentage of core layer in the wall thickness decreases and the higher orientation and lower change degree of the fibers in the front half of the main cavity along the melt flow direction.

\section{Conclusion}

The numerical calculation is carried out by combining the viscoelastic constitutive equation White-Metzner and the fiber orientation model iARD-RPR and then verified by experiment. Firstly, the water penetration condition of the short-shot water-assisted injection molding with and without the overflow cavity is compared. From theory and common sense, the short-shot water-assisted injection molding with the overflow cavity is more advantageous than that without the overflow cavity. And then, during short-shot waterassisted injection molding with the overflow cavity, the effects of the melt short shot size, water injection delay time, melt temperature, and water injection pressure on the penetration of water after penetration and the orientation distribution of short fibers along the melt flow direction were analyzed.

It is known that the melt short shot size has the most significant influence on the penetration condition and the orientation distribution of short fibers along the melt flow direction after water penetration, followed by the water injection pressure, the water injection delay time, and finally, the melt temperature. To a certain extent, as the melt short shot size increases, the residual wall thickness becomes larger after water penetration, and the orientation of short fibers is lower and the degree of the fiber orientation change is higher along the melt flow direction; as the water injection pressure increases, the residual wall thickness becomes thinner after water penetration, and the orientation of short fibers is higher (especially in the front half of the main cavity) and the degree of the fiber orientation change is lower along the melt flow direction; as the water injection delay time is prolonged, the residual wall thickness becomes thicker after water penetration, and the orientation of short fibers is lower and the degree of the fiber orientation change is higher along the melt flow direction in the front half of the main cavity; however, the penetration of the water and the orientation distribution of short fibers along the melt flow direction in the latter half of the main cavity have little effect; as the melt temperature increases, the residual wall thickness becomes thinner after water penetration, and the orientation of short fibers is higher and the degree of the fiber orientation change is lower along the melt flow direction in the front half of the main cavity, and the influence of the water penetration and the orientation distribution of short fibers along the melt flow direction in the latter half of the main cavity is minimal.

\section{Data Availability}

All data included in this study are available upon request by contact with the corresponding author.

\section{Conflicts of Interest}

The authors declare that they have no conflicts of interest.

\section{Acknowledgments}

This work forms part of a project supported by the National Natural Science Foundation of China (NSFC, No. 21664002, No. 51563010, and No. 51403165) and the Science and Technology Association of Jiangxi Province (No. GJJ161049).

\section{References}

[1] M. Knights, "Water injection molding makes hollow parts faster," Plastics Technology, vol. 48, no. 4, pp. 42-47, 2002.

[2] T. Juntgen and W. Michaeli, "The water injection technique(WIT) as an attractive alternative and supplement to gasassisted injection molding (GAIM)," in ANTEC 2002Conference Proceedings, pp. 386-391, San Fransisco, CA, USA, 2002.

[3] S.-J. Liu, "Water assisted injection molding: a review," International Polymer Processing, vol. 24, no. 4, pp. 315-325, 2009.

[4] H. X. Huang and Z. W. Deng, "Effects and optimization of processing parameters in water-assisted injection molding," Journal of Applied Polymer Science, vol. 108, no. 1, pp. 228 235, 2008.

[5] Z. Zeng-meng, Z. Hua, Y.'a. Gao, and H. Yang, "Simulation simulation and analysis on cavity filling process in waterassisted injection molding," Journal of Mechanical Engineering, vol. 46, no. 8, pp. 140-146, 2010.

[6] K. Zhang, T. Kuang, H. Liu, X. Zeng, Y. Deng, and H. Jasak, "Simulation analysis of water-assisted injection filling process based on viscoelastic constitutive," Polymer Materials Science and Engineering, vol. 30, no. 9, pp. 93-96, 2014.

[7] A. Polynkin, L. Bai, J. F. T. Pittman et al., "Water assisted injection moulding: development of insights and predictive capabilities through experiments on instrumented process in parallel with computer simulations," Plastics, Rubber and Composites, vol. 37, no. 2-4, pp. 131-141, 2008.

[8] T. Pudpong, P. Buahom, S. Areerat, W. Rungseesantivanon, I. Satoh, and T. Saito, "The effects of processing parameters on the residual wall thickness distribution at the sharp angle corner of water assisted injection molded parts," International Polymer Processing, vol. 28, no. 5, pp. 528-540, 2013.

[9] H. Park, B. S. Cha, and B. Rhee, "Experimental and Numerical Investigation of the Effect of Process Conditions on Residual Wall Thickness and Cooling and Surface Characteristics of Water- Assisted Injection Molded Hollow Products," Advances in Materials Science and Engineering, vol. 2015, Article ID 161938, 11 pages, 2015.

[10] T. Kuang, C. Yu, B. Xu, and L.-S. Turng, "Experimental study of penetration interfaces in the overflow fluid-assisted co- 
injection molding process," Journal of Polymer Engineering, vol. 36, no. 2, pp. 139-148, 2016.

[11] T.-Q. Kuang, K. Zhou, L.-X. Wu, G.-F. Zhou, and L.-S. Turng, "Experimental study on the penetration interfaces of pipes with different cross-sections in the overflow water-assisted coinjection molding," Journal of Applied Polymer Science, vol. 132, 2016.

[12] X. Liu, Y. Pan, G. Zheng et al., "Overview of the experimental trends in water-assisted injection molding," Macromolecular Materials and Engineering, vol. 303, no. 8, article 1800035, 2018.

[13] B. Wang, H.-x. Huang, and Z.-y. Wang, "Formation mechanism of transcrystals in PP/SAN blends prepared via waterassisted injection molding," Acta Polymerica Sinica, vol. 8, pp. 825-830, 2012.

[14] X. Liu, C. Zhang, K. Dai, G. Zheng, C. Liu, and C. Shen, "Unexpected molecular weight dependence of shish kebab in waterassisted injection molded HDPE," Polymers for Advanced Technologies, vol. 24, no. 2, pp. 270-272, 2013.

[15] X. Liu, Y. Pan, C. Peng et al., "Twisted lamellae in waterassisted injection molded high density polyethylene," Materials Letters, vol. 172, no. 1, pp. 19-22, 2016.

[16] H. Zhou, H. Liu, T. Kuang, Q. Jiang, Z. Chen, and W. Li, "Simulation and optimization of short fiber circumferential orientation in short-fiber-reinforced composites overflow waterassisted injection molded tube," Advances in Polymer Technology, vol. 2019, Article ID 6135270, 10 pages, 2019.

[17] H.-C. Tseng, R.-Y. Chang, and C.-H. Hsu, "Phenomenological improvements to predictive models of fiber orientation in concentrated suspensions," Journal of Rheology, vol. 57, no. 6, pp. 1597-1631, 2013. 